\section{Priorities in Palliative Care Research II}

\author{
Feature Editor: R. Sean Morrison
}

\title{
Introduction to Series
}

\author{
R. Sean Morrison, $\mathrm{MD}^{1}$ and Susan Zieman, $\mathrm{MD}, \mathrm{PhD}^{2}$
}

$\mathbf{F}$ IVE YEARS AGO, a diverse group of palliative care investigators met in Bethesda, Maryland, under the auspices of the National Institute on Aging and the National Palliative Care Research Center (NPCRC) to survey the evidence base of palliative care as it pertained to older adults and identify a series of research priorities for the field. Those priorities, published as a series in the Journal of Palliative Medicine $(J P M)$ in 2013, have helped shape funding announcements at both National Institutes of Health (NIH) and NPCRC and additionally have influenced the National Alzheimers Project and the Patient Centered Outcomes Research Center research initiatives. Recognizing the workforce constraints within the palliative care research community and building upon clinical work that has focused on ensuring that the core knowledge and skills of palliative care are inserted in a variety of specialties and settings, NPCRC and NIH convened a follow-up meeting in Bethesda, MD to identify palliative care research priorities in four additional key areas-heart disease, pulmonary and critical care, chronic kidney disease, and surgery. Beginning with this month's journal, articles summarizing the current state of the science and agreements for research priorities will be published both by JPM and concurrently by an appropriate specialty journal.
Why the focus on identifying key priorities? Federally funded research in the United States has been driven largely by investigator-initiated applications responding to broad topic areas. In the presence of an extensive evidence base, a large community of researchers, and abundant funding, this approach has fostered and delivered innovative and groundbreaking scientific discoveries. However, given increased competition for research funding, modest evidence to support palliative care clinical practice, and the limited but growing number of interdisciplinary researchers, a more strategic and focused approach to research is needed that will benefit seriously ill patients and their families. It is our hope that the questions and approaches identified in the NPCRC/NIH workshop and described in these upcoming articles will serve as a roadmap for both funders and researchers.

Address correspondence to: R. Sean Morrison, MD National Palliative Care Research Center Icahn School of Medicine at Mount Sinai Box 1070

New York, NY 10029

E-mail: sean.morrison@mssm.edu

${ }^{1}$ Brookdale Department of Geriatrics, Hertzberg Palliative Care Institute, Icahn School of Medicine at Mount Sinai, New York, New York. ${ }^{2}$ National Institute on Aging, Bethesda, Maryland. 